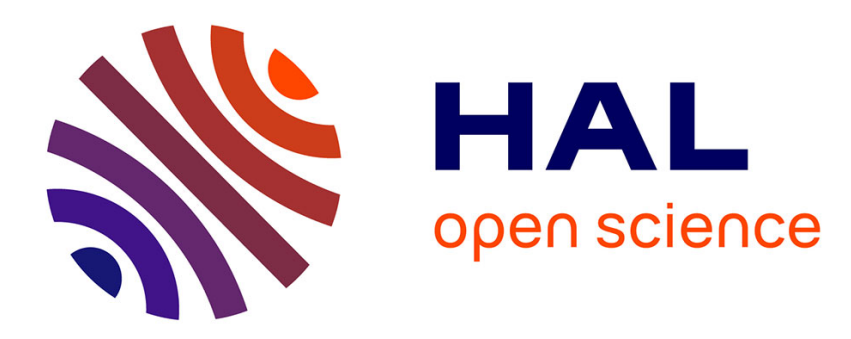

\title{
Lyapunov-Krasovskii Functionals Parameterized with Polynomials
}

\author{
Alexandre Seuret
}

\section{To cite this version:}

Alexandre Seuret. Lyapunov-Krasovskii Functionals Parameterized with Polynomials. ROCOND 2009 - 6th IFAC Symposium on Robust Control Design, Jun 2009, Haifa, Israel. 10.3182/200906163-IL-2002.00037 . hal-00386275

\section{HAL Id: hal-00386275 https://hal.science/hal-00386275}

Submitted on 20 May 2009

HAL is a multi-disciplinary open access archive for the deposit and dissemination of scientific research documents, whether they are published or not. The documents may come from teaching and research institutions in France or abroad, or from public or private research centers.
L'archive ouverte pluridisciplinaire HAL, est destinée au dépôt et à la diffusion de documents scientifiques de niveau recherche, publiés ou non, émanant des établissements d'enseignement et de recherche français ou étrangers, des laboratoires publics ou privés. 


\title{
Lyapunov-Krasovskii Functionals Parameterized with polynomials
}

\author{
Alexandre Seuret ${ }^{*, 1}$ \\ * Automatic Control Department of Grenoble GIPSA-Lab, UMR CNRS \\ 5216, BP. 46, F-38402, Saint Martin d'Hères, France. \\ E-mail: alexandre.seuret@gipsa-lab.inpg.fr
}

\begin{abstract}
A novel method based on Lyapunov-Krasovskii functionals for the stability analysis of linear systems with constant is introduced. The Lyapunov-Krasovskii functionals are provided using polynomial parameters. Stability conditions are derived in the form of linear matrix inequalities. Examples show that these computationally tractable conditions can give tighter stability results than the ones in the literature.
\end{abstract}

Keywords: Time-delay systems; Stability analysis; Lyapunov-Krasovskii functionals; Linear matrix inequalities

\section{INTRODUCTION}

Stability analysis of time-delay systems is an important topic in many disciplines of science and engineering $\mathrm{Gu}$ et al. [2003], Niculescu [2001], Richard [2003]. Motivating applications are found in diverse areas, such as biology, chemistry, tele-communication control engineering, economics, and population dynamics Kolmanovskii and Myshkis [1999]. There has been an increased interest in the area of time-delay systems over the last two decades due to the emerging area of networked embedded systems, which are systems where sensor and actuator devices communicate with control nodes over a communication network. In such systems, processing time in the network nodes together with propagation delays in the inter-node communication lead necessarily to time delays affecting the overall closed-loop control system. Various phenomena related to delays in networked controlled systems have recently been considered, e.g., packet losses Naghshtabrizi et al. [December 13-15, 2006], Hespanha et al. [2007] and robust sampling Fridman et al. [2004].

A lot of attention has been dedicated to the LyapunovKrasovskii theory for the analysis of time-delay systems Richard [2003], Niculescu [2001], Gu et al. [2003]. The emergence of semi-definite programming Boyd et al. [1994] allows solving the stability conditions, usually expressed as Linear Matrix Inequalities (LMI). In the literature, several types of Lyapunov-Krasovskii functionals (LKFs) have been proposed, leading to different level of conservatism. In the case of LKFs with constant parameters, model transformations can reduce the conservatism Ariba and Gouaisbaut [2007a], Fridman and Shaked [2002, 2006], Gu and Niculescu [2000], He et al. [2007], Kao and Rantzer [2005], Wu et al. [2004]. Stability conditions are generally constrained by the choice of the constant parameters of the LKFs which considerably increase the conservatism. There exist systems that are not stable for small delays

\footnotetext{
1 The work by A. Seuret is supported by the European Commission through the FP7 Project FeedNetBack.
}

but become stable for sufficiently large delays. Physical examples exists in high-speed networks, biological systems and other areas Kolmanovskii and Myshkis [1999]. The stability of such systems cannot be shown by LKFs with constant parameters. The introduction of more complex and flexible LKFs is required to overcome these difficulties. The discretization method introduced in $\mathrm{Gu}$ [1997], Gu et al. [2003], allows constructing piecewise linear functions as the LKF parameters by dividing the delay interval into several smaller intervals on which the parameters of the LKF are linearly varying. The stability analysis leads to less conservative conditions than in the case of constant parameters but extensions to the time-varying delay case are not straightforward. In Papachristodoulou et al. [Dec. 12-14, 2007], a method to build LKFs with varying parameters based on sum of squares tools is introduced. However the computational complexity is quite high. Another recent approach method is based on Integral Quadratic Constraints (IQC) Kao and Rantzer [2007]. It provides a new interpretation of the LKF and the potential conservatism (see Ariba and Gouaisbaut [2007b]). In Zhabko [2001], complete LKFs, which correspond to necessary and sufficient conditions of stability, are constructed by solving a functional differential equation. It is useful to derive robust stability conditions with respect to delay variations Fridman and Niculescu [2008] or parameter uncertainties Kolmanovskii and Zhabko [2003], Mondie et al. [2005].

In this article, a novel method to parameterize LKFs is provided. The parameters are polynomial functions. The candidate of the LKF is the same as the one defined in Papachristodoulou et al. [Dec. 12-14, 2007]. However in this article, the stability conditions only concerns constant delay and are provided using LMIs but not sum of squares tools. The parameters are defined using a polynomial solution of a particular linear differential equation. The resulting stability condition is expressed in terms of LMIs. The method is able to handle systems with constant delays. 
The paper is organized as follows: Section II is devoted to the formulation of the problem. The form of the LKFs is examined in Section III. Section IV concerns the stability analysis in the case of constant delays. Two examples are provided in Section VI to show the efficiency of the method. The paper is concluded in Section VII.

Notations. Given an $n$-dimensional state vector $x$ and a non-negative delay $\tau, x_{t}$ denotes a function such that $x_{t}(\theta)=x(t-\theta)$ for all $\theta \in[-\tau, 0]$. For a matrix $P \in \mathbb{R}^{n \times n}, P^{T}$ denotes matrix transposition and $P>0$ denotes that $P=P^{T}$ is positive definite. The $n \times n$ identity matrix is denoted $I_{n}$. For any matrices $A$ and $B, \operatorname{diag}(A, B)$ denotes the block diagonal matrix with $A$ and $B$ on the diagonal and zeros elsewhere. The notation $A_{k, i j}$ indicates the $k \times k$ matrix located between rows $i k+1$ and $i k+k$ and columns $j k+1$ and $j k+k$. An asterisk in a symmetric matrix denotes a matrix element that is from the corresponding upper triangular part. The symbole " $\otimes$ " represents the classical Kronecker product between two matrices.

\section{PROBLEM FORMULATION}

Consider a linear system with a single delay

$$
\left\{\begin{array}{l}
\dot{x}(t)=A_{0} x(t)+A_{1} x(t-\tau) \\
x(\theta)=\phi(\theta), \quad \forall \theta \in[-\tau, 0]
\end{array}\right.
$$

where $x(t) \in \mathbb{R}^{n}$ is the state variable and $A_{0}, A_{1} \in \mathbb{R}^{n \times n}$ are constant matrices. The delay $\tau>0$ is assumed to be constant. The function $\phi:[-\tau, 0] \rightarrow \mathbb{R}^{n}$ represents the function of the initial conditions. We use the general form of LKFs of $\mathrm{Gu} \mathrm{Gu}$ [1997]

$$
\begin{aligned}
V\left(x_{t}\right)= & x^{T}(t) P x(t)+2 x^{T}(t) \int_{-\tau}^{0} Q(\xi) x(t+\xi) d \xi \\
& +\int_{-\tau}^{0} x^{T}(t+\xi) S(\xi) x(t+\xi) d \xi \\
& +\int_{-\tau}^{0} \int_{-\tau}^{0} x^{T}(t+s) R(s, \xi) d s x(t+\xi) d \xi
\end{aligned}
$$

where $P \in \mathbb{R}^{n \times n}$ is positive definite and $Q(\xi), R(s, \xi)=$ $R^{T}(\xi, s), S(\xi) \in \mathbb{R}^{n \times n}$ are continuous functions.

The problem considered in this paper is how to design LKFs (2) with polynomial parameters. As noted in the introduction, interesting developments already allow considering variations in $Q, R$ and $S$. Developments have been provided to construct piecewise constant (Ariba and Gouaisbaut [2007b], Kao and Rantzer [2007]), piecewise linear parameters (Gu [1997], Gu et al. [2003]) and polynomials parameters (Papachristodoulou et al. [Dec. 12-14, 2007]). This article introduces a novel method which allows an explicit construction of polynomial functions $Q, R$ and $S$ of any order. The method is based on the solutions of a linear differential equation. The stability conditions are given in terms of linear matrix inequalities.

\section{PARAMETRIZATION OF LYAPUNOV-KRASOVSKII FUNCTIONALS}

As in Seuret and Johansson [2009], we consider some real functions $f^{i}$ where $i=1, \ldots, N$ and the following functions $Q, R$ and $S$ such that for all $s$ and $\xi$ in $[-\tau, 0]$

$$
\begin{aligned}
& Q(\xi)=\sum_{i=1}^{N} f^{i}(\xi) Q_{i}, \\
& S(\xi)=\sum_{i=1}^{N} \sum_{j=1}^{N} f^{i}(\xi) f^{j}(\xi) S_{i j}, \\
& R(s, \xi)=\sum_{i=1}^{N} \sum_{j=1}^{N} f^{i}(s) f^{j}(\xi) R_{i j}
\end{aligned}
$$

where $Q_{i}, S_{i j}$ and $R_{i j}$ for $i, j=1, \ldots, N$ are constant matrices. Introducing the vector function $W_{f}(\xi)=$ $\left[f^{1}(\xi) \ldots f^{N}(\xi)\right]^{T}$, a nice expression of the functions can be derived

$$
\begin{aligned}
& Q(\xi)=\mathcal{Q W}_{f}(\xi), \\
& S(\xi)=\mathcal{W}_{f}^{T}(\xi) \mathcal{S} \mathcal{W}_{f}(\xi), \\
& R(s, \xi)=\mathcal{W}_{f}^{T}(s) \mathcal{R} \mathcal{W}_{f}(\xi)
\end{aligned}
$$

where $\mathcal{W}_{f}=W_{f} \otimes I_{n}$ and the constant matrices $\mathcal{Q}_{f}, \mathcal{R}_{f}$ and $\mathcal{S}_{f}$ are such that $\left(\mathcal{Q}_{f}\right)_{n, i 1}=Q_{i},\left(\mathcal{R}_{f}\right)_{n, i j}=R_{i j}$ and $\left(\mathcal{S}_{f}\right)_{n, i j}=S_{i j}$. The functions which defined the LKF are thus expressed in a simple way. A lemma to ensure that the LKF is positive definite is thus formulated.

Lemma 1. (Seuret and Johansson [2009]) Consider a given delay $\tau>0$. If there exist two positive definite matrices $P \in \mathcal{R}^{n \times n}$ and $\mathcal{S} \in \mathbb{R}^{N n \times N n}$ and two matrices $\mathcal{Q} \in \mathbb{R}^{n \times N n}$ and $\mathcal{R} \in \mathbb{R}^{N n \times N n}$ such that

$$
\Xi=\left[\begin{array}{cc}
P & \mathcal{Q} \\
* & \mathcal{R}+\mathcal{S} / \tau
\end{array}\right]>0
$$

Then the functional $V$ with (3) is positive definite.

Proof. Consider the functional (2) with the functions $Q, R, S$ as in (3). Define the vector $\Phi_{f}(t)=\int_{-\tau}^{0} W_{f}(\xi) x(t+$ $\xi) d \xi$. The second and the last terms of $V$ can thus be rewritten as $2 x^{T}(t) \mathcal{Q} \Phi_{f}(t)$ and $\Phi_{f}^{T}(t) \mathcal{R} \Phi_{f}^{T}(t)$. Provided that $\mathcal{S}>0$, Jensen's inequality ensures that $\int_{-\tau}^{0} x^{T}(t+$ $\xi) W_{f}^{T}(\xi) \mathcal{S} W_{f}(\xi) x(t+\xi) d \xi \geq \Phi_{f}^{T}(t) \mathcal{S} / \tau \Phi_{f}(t)$. Denote $\xi_{0}(t)=\left[x^{T}(t), \Phi_{f}^{T}(t)\right]^{T}$, the functional satisfies $V\left(x_{t}\right) \geq$ $\xi_{0}^{T}(t) \Xi \xi_{0}(t) \geq \epsilon\|x\|^{2}$ and $V$ is positive definite if (4) holds.

Remark 1. Inequality (4) which concerns the positive definiteness of the LFK is the same as in Gu [1997]. Lemma 1 is an extension of the result from $\mathrm{Gu}$ [1997] to all LKFs defined as (2) with (3).

In Lemma 1, additional informations are taken into account to design less conservative conditions. In Ariba and Gouaisbaut [2007a,b], it was proven that increasing the dimension of the state vector (considering $X_{1}=$ $\left[x^{T}(t) \dot{x}^{T}(t) \ldots\left(x^{(N)}\right)^{T}(t)\right]^{T}$ or $X_{2}=\left[x^{T}(t) x^{T}(t-\tau / N)\right.$ $\left.\left.\ldots x^{T}(t-\tau)\right]^{T}\right)$ considerably reduces the conservatism. In this article, we introduce the vector $\Phi_{f}$ with polynomial functions $f^{i}$.

\subsection{A simple method to produce the LKF parameters}

As in Seuret and Johansson [2009], for a given integer $N$, consider a matrix $D \in \mathbb{R}^{N \times N}$. Define $W:[-\tau, 0] \rightarrow \mathbb{R}^{N}$ such that

$$
\left\{\begin{array}{l}
\dot{W}(\xi)=D W(\xi) \\
W(0)=W_{0}
\end{array}\right.
$$


where $W_{0} \in \mathbb{R}^{N}$. Consider now the LKF (2) defined by

$$
\begin{gathered}
Q(\xi)=\mathcal{Q} \mathcal{W}(\xi), \\
S(\xi)=\mathcal{W}^{T}(\xi) \mathcal{S} \mathcal{W}(\xi), \\
R(s, \xi)=\mathcal{W}^{T}(s) \mathcal{R} \mathcal{W}(\xi)
\end{gathered}
$$

where $\mathcal{W}=W \otimes I_{n}, \mathcal{Q}$ is in $\mathbb{R}^{n \times n N}$ and $\mathcal{S}, \mathcal{R} \in \mathbb{R}^{n N \times n N}$ are symmetric constant matrices. In the latter, we will say that the pair $\left(D, W_{0}\right)$ generates the LKF $V$ if the functions $Q, R$ and $S$ are given by (5) and (6). There is no restriction on the matrix $D$. Depending on its eigenvalues, $Q, R$ and $S$ can be polynomial, exponential and trigonometric functions. As $W$ is the solution of the linear differential equations of the type of (5), the functions $Q, R$ and $S$ are infinitely differentiable over the interval $[-\tau, 0]$. As the vector function satisfies a linear differential equations, the functions $Q, R$ and $S$, their derivative could be easily derived.

Lemma 2. The differentiation of the functions $Q, S, R$ are given by

$$
\begin{aligned}
& \dot{Q}(\xi)=\mathcal{Q D} \mathcal{W}(\xi) \\
& \dot{S}(\xi)=\mathcal{W}^{T}(\xi)\left[\mathcal{D}^{T} \mathcal{S}+\mathcal{S} \mathcal{D}\right] \mathcal{W}(\xi) \\
& \frac{\partial R(s, \xi)}{\partial s}=\frac{\partial R^{T}(s, \xi)}{\partial \xi}=\mathcal{W}^{T}(s) \mathcal{D}^{T} \mathcal{R} \mathcal{W}(\xi)
\end{aligned}
$$

and their evaluation at any instant $a$ and $b$ in $[-\tau, 0]$ are as follows

$$
\begin{aligned}
& Q(a)=\mathcal{Q} e^{a \mathcal{D}} \mathcal{W}_{0}, \\
& S(a)=\mathcal{W}_{0}^{T}\left(e^{a \mathcal{D}}\right)^{T} \mathcal{S} e^{a \mathcal{D}} \mathcal{W}_{0}, \\
& R(a, b)=\mathcal{W}_{0}^{T}\left(e^{a \mathcal{D}}\right)^{T} \mathcal{R} e^{b \mathcal{D}} \mathcal{W}_{0} .
\end{aligned}
$$

where $\mathcal{D}=D \otimes I_{n}$ and $\mathcal{W}_{0}=W_{0} \otimes I_{n}$.

To obtain polynomial parameters, the matrix $D$ should be a nilpotent matrix, i.e. there exists a integer $N^{*} \leq N$ such that $D^{N^{*}}=0$. In the latter, we will only consider the matrix

$$
D=\left[\begin{array}{ccccc}
0 & 0 & \ldots & 0 & 0 \\
1 & 0 & \ldots & 0 & 0 \\
\vdots & \ddots & \ddots & \vdots & \vdots \\
0 & 0 & \ldots & 0 & 0 \\
0 & 0 & \ldots & N-1 & 0
\end{array}\right] \quad \text { and } W_{0}=\left[\begin{array}{c}
1 \\
0 \\
\vdots \\
0 \\
0
\end{array}\right]
$$

In such situation, the vector function $W(\xi)$ is given by:

$$
W(\xi)=e^{D \xi} W_{0}=\left[\begin{array}{c}
1 \\
\vdots \\
\xi^{N-1}
\end{array}\right] .
$$

It is thus easy to see that the parameters $Q, R$ and $S$ are polynomial functions. These parameters are defined by the constant matrices $\mathcal{Q}, \mathcal{R}$ and $\mathcal{S}$ which allow defining any polynomials of degree $N-1$ or $2 N-2$. In the next section, a stability criteria is provided based on such parameters.

It is clear that if one considers other nilpotent matrices $D$ and/or other vectors $W_{0}$, the results will be equivalent since the main objective is to define parameter for the LKF. The use of various and different pairs of $\left(D, W_{0}\right)$ just corresponds to a change of coordinates of the polynomial.

In the sequel, the variable $\Phi(t)$ is used to represent

$$
\Phi(\xi)=\int_{-\tau}^{0} W(\xi) x(t+\xi) d \xi=\left[\begin{array}{c}
\int_{-\tau}^{0} \xi^{0} x(t+\xi) d \xi \\
\vdots \\
\int_{-\tau}^{0} \xi^{N-1} x(t+\xi) d \xi
\end{array}\right]
$$

Note that using integrations by parts, it is possible to see the vector $\Phi(t)$ as an augmented vector of the primitives of the state $x$.

\section{STABILITY ANALYSIS}

In this section, the stability analysis of systems with constant delay is provided. Based on functionals of the form (2) with (6), easy computable LMIs are developed. Consider system (1) with a constant delay $\tau$. The following theorem holds:

Theorem 1. For a given $N$ and a constant delay $\tau>0$. If there exist $P=P^{T} \in \mathbb{R}^{n \times n}, \mathcal{Q} \in \mathbb{R}^{n \times n N}$ and $\mathcal{S}, \mathcal{T}$, $\mathcal{R}=\mathcal{R}^{T} \in \mathbb{R}^{n N \times n N}$ such that

$$
\mathcal{S}>0, \quad \mathcal{T}>0, \quad \Xi>0,
$$

$$
\Pi_{1}=\mathcal{T}+\mathcal{D}^{T}(\mathcal{S}+\tau \mathcal{T})+(\mathcal{S}+\tau \mathcal{T}) \mathcal{D}+\mathcal{F} \mathcal{T}+\mathcal{T} \mathcal{F}>0
$$

and

$$
\Pi_{2}=\left[\begin{array}{ccc}
\pi_{11} & P A_{1}-\mathcal{Q} \mathcal{W}(-\tau) & A_{0}^{T} \mathcal{Q}+\mathcal{W}_{0}^{T} \mathcal{R}-\mathcal{Q D} \\
* & -\mathcal{W}(-\tau)^{T} \mathcal{S W}(-\tau) & A_{1}^{T} \mathcal{Q}-\mathcal{W}^{T}(-\tau) \mathcal{R} \\
* & * & \pi_{44}
\end{array}\right]<0
$$

where

$$
\begin{aligned}
& \pi_{11}=P A_{0}+A_{0}^{T} P+\mathcal{Q} \mathcal{W}_{0}+\left(\mathcal{Q} \mathcal{W}_{0}\right)^{T}+\mathcal{W}_{0}^{T} \mathcal{S} \mathcal{W}_{0}, \\
& \pi_{44}=-\Pi_{1} / \tau-\mathcal{D}^{T} \mathcal{R}+\mathcal{R} \mathcal{D}, \\
& \mathcal{F}=\operatorname{diag}\{0,1, \ldots, N-1\} \otimes I_{n}
\end{aligned}
$$

Then system (1) is asymptotically stable.

Proof. Consider an integer $N$ and the matrix $D \in \mathbb{R}^{N \times N}$, $W_{0} \in \mathbb{R}^{N}$ as defined in (7). The functional is rewritten as

$$
\begin{aligned}
V\left(x_{t}\right)= & x^{T}(t) P x(t)+2 x^{T}(t) \int_{-\tau}^{0} Q(\xi) x(t+\xi) d \xi \\
& +\int_{-\tau}^{0} x^{T}(t+\xi) S(\xi) x(t+\xi) d \xi \\
& +\int_{-\tau}^{0} \int_{-\tau}^{0} x^{T}(t+s) R(s, \xi) d s x(t+\xi) d \xi
\end{aligned}
$$

where $Q, R$ are given in (6) and

$$
S(\xi)=\mathcal{W}^{T}(\xi) \mathcal{S W}(\xi)+(\xi+\tau) \mathcal{W}^{T}(\xi) \mathcal{T} \mathcal{W}(\xi)
$$

where $\mathcal{S}$ and $\mathcal{T} \in \mathbb{R}^{n N \times n N}$ are constant matrices. Denote $\xi_{0}(t)=\left[x^{T}(t), \Phi^{T}(t)\right]^{T}$, it is easy to see that

$$
\begin{aligned}
V\left(x_{t}\right) & \geq \xi_{0}^{T}(t) \Xi \xi_{0}(t) \\
& +\int_{-\tau}^{0} \int_{-\tau}^{0} x^{T}(t+\xi)(\xi+\tau) \mathcal{W}^{T}(\xi) \mathcal{T} \mathcal{W}(\xi) x(t+\xi) d \xi
\end{aligned}
$$

Then if $\Xi>0$ and $\mathcal{T}>0, V$ is positive definite. The second part of the proof concerns the analysis of the derivative of the functional $V$. Using an integration by parts, the derivative of $\Phi(t)$, denoted as $\Phi_{i}(t)=\int_{-\tau}^{0} \xi^{i-1} x(t+\xi) d \xi$, is given by 


$$
\begin{aligned}
\dot{\Phi}(\xi) & =\left[\begin{array}{l}
\dot{\Phi}_{0}(t) \\
\dot{\Phi}_{1}(t) \\
\vdots \\
\dot{\Phi}_{N-1}(t)
\end{array}\right]=\left[\begin{array}{l}
x(t)-x(t-\tau) \\
-(-\tau) x(t-\tau)-\Phi_{0}(t) \\
\vdots \\
-(-\tau)^{N-1} x(t-\tau)-\Phi_{N-2}(t)
\end{array}\right] \\
& =-\mathcal{D} \Phi(t)+\mathcal{W}(0) x(t)-\mathcal{W}(-\tau) x(t-\tau) .
\end{aligned}
$$

As in $\mathrm{Gu}$ et al. [2003], the differentiation of $V$ leads to

$$
\begin{aligned}
\dot{V}\left(x_{t}\right) & =2 \dot{x}^{T}(t) P x(t)+x^{T}(t)\left(Q W(0)+Q^{T}(0)+S(0)\right) x(t) \\
& -2 x^{T}(t) Q(-\tau) x(t-\tau)-x(t-\tau) S(-\tau) x(t-\tau) \\
& -2 x^{T}(t-\tau) \int_{-\tau}^{0} R(0, \xi) x(t+\xi) d \xi \\
& +2 \dot{x}^{T}(t) \int_{-\tau}^{0} Q(\xi)(t+\xi) d \xi \\
& +2 x^{T}(t) \int_{-\tau}^{0}[Q \mathcal{D}+R(0, \xi)] x(t+\xi) d \xi \\
& -\int_{-\tau}^{0} x^{T}(t+\xi) \dot{S}(\xi) x(t+\xi) d \xi-\int_{-\tau}^{0} \int_{-\tau}^{0} x^{T}(t+s) \\
& \left(\mathcal{D}^{T} R(s, \xi)+R(s, \xi) \mathcal{D}\right) x(t+\xi) d \xi d s
\end{aligned}
$$

From the definition of $S$, its derivative is given by

$$
\begin{aligned}
\dot{S}(\xi)= & \mathcal{W}^{T}(\xi)\left((\mathcal{S}+\tau \mathcal{T}) \mathcal{D}^{T}+\mathcal{D}(\mathcal{S}+\tau \mathcal{T})+T\right) \mathcal{W}(\xi) \\
& +2 \xi \mathcal{W}(\xi) \mathcal{D} \mathcal{T} \mathcal{W}(\xi)
\end{aligned}
$$

Noting that the last term can be rewritten as follows

$$
\begin{aligned}
2 \xi \mathcal{W}^{T}(\xi) \mathcal{T} \mathcal{D} \mathcal{W}(\xi) & =2 \mathcal{W}^{T}(\xi) \mathcal{T} \mathcal{D}\left[\begin{array}{lll}
\xi & \ldots & \xi^{N}
\end{array}\right]^{T} \\
& =2 \mathcal{W}^{T}(\xi) \mathcal{T}\left[\begin{array}{llll}
0 & D
\end{array}\right]\left[\begin{array}{lll}
1 & \ldots & \xi^{N}
\end{array}\right]^{T} \\
& =2 \mathcal{W}^{T}(\xi) \mathcal{T} \mathcal{F} \mathcal{W}(\xi)
\end{aligned}
$$

Replacing $\dot{x}(t)$ by $A_{0} x(t)+A_{1} x(t-\tau)$ and applying Lemma 2, (12) becomes

$$
\begin{aligned}
\dot{V}\left(x_{t}\right) & =x^{T}(t)\left(P A_{0}+A_{0}^{T} P+\mathcal{Q} \mathcal{W}_{0}+\mathcal{W}_{0}^{T} \mathcal{Q}^{T}\right. \\
& \left.+\mathcal{W}_{0}^{T} \mathcal{S} \mathcal{W}_{0}\right) x(t)+2 x^{T}(t)\left(P A_{1}-\mathcal{Q} \mathcal{W}(-\tau)\right) x(t-\tau) \\
& +2 x^{T}(t-\tau)\left(A_{1}^{T} \mathcal{Q}-\mathcal{W}^{T}(-\tau) \mathcal{R}\right) \Phi(t) \\
& -x^{T}(t-\tau) W^{T}(-\tau) \mathcal{S} W(-\tau) x(t-\tau) \\
& +2 x^{T}(t)\left[A_{0}^{T} \mathcal{Q}-\mathcal{Q D}+\mathcal{W}_{0}^{T} \mathcal{R}\right] \Phi(t) \\
& -\Phi^{T}(t)\left[\mathcal{D}^{T} \mathcal{R}+\mathcal{R} \mathcal{D}\right] \Phi(t) \\
& -\int_{-\tau}^{0} x^{T}(t+\xi) \mathcal{W}^{T}(\xi) \Pi_{1} \mathcal{W}(\xi) x(t+\xi) d \xi
\end{aligned}
$$

Since $\Pi_{1}>0$, Jensen's inequality ensures that the last term of the previous expression is bounded by $-\Phi^{T}(t) \Pi_{1} / \tau \Phi(t)$. Introducing the vector $\zeta(t)=\left[x^{T}(t)\right.$, $\left.x^{T}(t-\tau), \Phi^{T}(t)\right]^{T}$, one has $\dot{V}\left(x_{t}\right) \leq \zeta^{T}(t) \Pi_{2} \zeta(t)$. Then provided that $\Pi_{2}<0$, the derivative of the LKF $V$ is negative definite and, consequently, the system is asymptotically stable.

Remark 2. The number, $N^{*}$, from the discretization method of $\mathrm{Gu}$ [1997] and the dimension, $N$, of the matrix $D$ do not corresponds to the same level of complexity. In term of the quantity of variables to determine, a discretization of order $N^{*}$ is equivalent to solve Theorem 1 with $D$ of dimension $N+1$.

Remark 3. Theorem 1 provides only sufficient but not necessary conditions. The only conservatism introduced in the proof comes from the Jensen's inequality. However the increase of the dimension of $N$ reduces the conservatism.

Remark 4. The introduction of the matrice $\mathcal{T}$ is required to solve the stability conditions. If $\mathcal{T}$ is taken as the zero matrice, then $\Pi_{1}$ can not be definite positive and the last diagonal term of $\Pi_{2}$ can not be definite negative. This comes from the matrices $D$ which is a singular.

Remark 5. In Theorem 1, the number of variables to solve is $n(n+1) / 2+n^{2} N+3 n N(n N+1) / 2$. However there are redundancies in the variables. Since for each matrices matrices $R, S$ and $T$, the objective is to provide a polynomial parameters of degree $2 N-2$. Thus only $2 N-1$ matrix variables are required instead of $n N(n N+1) / 2$. Following this idea, it is possible to consider only matrices $\mathcal{R}$ ( and respectively $\mathcal{S}$ and $\mathcal{T}$ ) of the form:

$$
\left[\begin{array}{cccccc}
R_{11} & R_{12} & 0 & \ldots & 0 & 0 \\
R_{12}^{T} & R_{22} & R_{23} & \ldots & 0 & 0 \\
0 & R_{23}^{T} & R_{33} & \ddots & 0 & 0 \\
\vdots & \vdots & \ddots & \ddots & \ddots & \vdots \\
0 & 0 & \ldots & \ddots & R_{(N-2)(N-2)} & R_{(N-2)(N-1)} \\
0 & 0 & \ldots & \ldots & R_{(N-2)(N-1)}^{T} & R_{(N-1)(N-1)}
\end{array}\right]
$$

Then the necessary number of variables becomes $n(n+$ 1) $/ 2+3 / 2\left(n^{2}+n\right) N+3 n^{2}(N-1)$. The number of variable is thus not increasing as $N^{2}$ but as $N$.

There also are another source of redundancy of variable. It comes from the definition of the parameter $S$. Some terms of the polynomial defined with $\mathcal{S}$ and $\mathcal{T}$ have the same degree. It would be possible to reduce the number of variables to solve but this will not be considered in this article.

Remark 6. The stability conditions of Theorems 1 are expressed in a simple way compare to the discretization method. This is due to the use of the high dimensional matrices $\mathcal{Q}, \mathcal{R}, \mathcal{S}$ and $\mathcal{T}$ in the LMIs. In $\mathrm{Gu}$ [1997], the conditions are expressed using each square matrices $\mathcal{Q}_{n, 1, j}$ and so on, which requires lots of definitions. Another interesting aspect of Theorem 1 is that the LMIs strictly have the same expression whatever degree, $N$, of the polynomial, the nilpotent matrices $D$ and the initial vector condition $W_{0}$.

\section{SYSTEMS WITH PARAMETER UNCERTAINTIES}

Consider now the linear system with constant delay and parameter uncertainties represented as polytopic uncertainties:

$$
\dot{x}(t)=\sum_{i=1}^{M} \lambda_{i}(t)\left\{A_{0 i} x(t)+A_{1 i} x(t-\tau)\right\}
$$

where $M$ is an integer and the scalar functions $\lambda_{i}$ are such that

$$
\forall t \geq 0, \quad \sum_{i=1}^{M} \lambda_{i}(t) \text { and } \forall i=1, \ldots, M, \quad \lambda_{i}(t) \geq 0
$$

The following theorem holds

Theorem 2. For a given $N$ and a constant delay $\tau>0$. If there exist $P=P^{T} \in \mathbb{R}^{n \times n}, \mathcal{Q} \in \mathbb{R}^{n \times n N}$ and $\mathcal{S}, \mathcal{T}$, $\mathcal{R}=\mathcal{R}^{T} \in \mathbb{R}^{n N \times n N}$ such that for all $i=1, \ldots, M$

$$
\begin{gathered}
\mathcal{S}>0, \quad \mathcal{T}>0, \quad \Xi>0, \\
\Pi_{1}=\mathcal{T}+\mathcal{D}^{T}(\mathcal{S}+\tau \mathcal{T})+(\mathcal{S}+\tau \mathcal{T}) \mathcal{D}+\mathcal{F} \mathcal{T}+\mathcal{T} \mathcal{F}>0
\end{gathered}
$$

and $\Pi_{2 i}=$ 


\begin{tabular}{|c||c|c|c|c|}
\hline Theorems & $\mathrm{N}$ & 2 & 3 & 4 \\
\hline \hline Gu et al. [2003] & $\tau_{\min }$ & 0.43 & 0.35 & 0.32 \\
Fridman [2006a] & $\tau_{\min }$ & 0.12 & 0.12 & 0.1003 \\
\hline Theorem 1 & $\tau_{\min }$ & 0.1006 & 0.100169 & 0.100169 \\
\hline \hline Gu et al. [2003] & $\tau_{\max }$ & 1.09 & 1.46 & 1.55 \\
Fridman [2006a] & $\tau_{\max }$ & 1.24 & 1.51 & 1.59 \\
\hline Theorem 1 & $\tau_{\max }$ & 1.45 & 1.67 & 1.713 \\
\hline
\end{tabular}

Table 1. Maximal allowable constant delays $\tau$ for Example 1

$$
\left[\begin{array}{ccc}
\pi_{11 i} & P A_{1 i}-\mathcal{Q} \mathcal{W}(-\tau) & A_{0 i}^{T} \mathcal{Q}+\mathcal{W}_{0}^{T} \mathcal{R}-\mathcal{Q D} \\
* & -\mathcal{W}(-\tau)^{T} \mathcal{S} \mathcal{W}(-\tau) & A_{1 i}^{T} \mathcal{Q}-\mathcal{W}^{T}(-\tau) \mathcal{R} \\
* & * & \pi_{44}
\end{array}\right]<0
$$

where

$$
\begin{aligned}
& \pi_{11 i}=P A_{0 i}+A_{0 i}^{T} P+\mathcal{Q} \mathcal{W}_{0}+\left(\mathcal{Q} \mathcal{W}_{0}\right)^{T}+\mathcal{W}_{0}^{T} \mathcal{S} \mathcal{W}_{0}, \\
& \pi_{44}=-\Pi_{1} / \tau-\mathcal{D}^{T} \mathcal{R}-\mathcal{R} \mathcal{D} \\
& \mathcal{F}=\operatorname{diag}\{0,1, \ldots, N-1\} \otimes I_{n}
\end{aligned}
$$

Then system (13) is asymptotically stable.

Proof. The proof follows the line of Theorem 1 and uses the convexity of the function $\lambda_{i}(t)$.

\section{EXAMPLES}

Example 1 Consider the following system Fridman [2006b], Gu [1997]

$$
\dot{x}(t)=\left[\begin{array}{cc}
0 & 1 \\
-2 & 0.1
\end{array}\right] x(t)+\left[\begin{array}{ll}
0 & 0 \\
1 & 0
\end{array}\right] x(t-\tau)
$$

An analytical analysis in $\mathrm{Gu}$ et al. [2003] indicates that the system is stable if the delay belong to the interval ]0.100168, 1.7178[. The stability of such a system can not be performed using simple LKFs Gu [1997], Fridman and Niculescu [2008]. Table 1 exposes the results obtained by Theorem 1. The conservatism is reduced as the dimension of the matrix increases. The results are less conservative than the discretization method for the same level of complexity (number of variables to solve). Table 1 also shows that it is not required to increase so much the dimension of $D$ to provide an accurate estimate of the stability interval.

Example 2 Consider system (1) Fridman and Shaked [2002], Gu [1997], Kao and Rantzer [2005] or Wu et al. [2004] with

$$
\dot{x}(t)=\left[\begin{array}{cc}
-2 & 0 \\
0 & -0.9
\end{array}\right] x(t)+\left[\begin{array}{cc}
-1 & 0 \\
-1 & -1
\end{array}\right] x(t-\tau)
$$

As $\tau$ is constant, an application of the Nyquist criterion shows that the system is stable has eigenvalues on the imaginary axis for $\tau=6.172$. The results from the literature and using Theorem 2 are summarized in Table 2. A first comment concerns the fact that when $N=1$, which finally corresponds to the case of constant parameters, the stability conditions are equivalent to the various methods which also consider constant parameters. Then for $N \geq$ 0 , the conservatism is reduced by the introduction of polynomials in the LKF's parameters. For this example, the results obtained by Theorem 1 are less conservative than using most of existing ones.

\begin{tabular}{|l|c|}
\hline Existing theorems & $\tau_{\max }$ \\
\hline \hline Fridman and Shaked [2002] & 4.472 \\
Wu et al. [2004] & 4.472 \\
Kao and Rantzer [2005] & 4.472 \\
He et al. [2007] & 4.472 \\
Ariba and Gouaisbaut [2007a] & 5,120 \\
Gu [1997] & 6.07 \\
Kao and Rantzer [2007] & 6.117 \\
Gu [2001] & 6.171 \\
Ariba and Gouaisbaut [2008], N=2 & 5.717 \\
Ariba and Gouaisbaut [2008], N=4 & 5.967 \\
Ariba and Gouaisbaut [2008], N=6 & 6.120 \\
\hline \hline Theorem 1, N=1 & 4.472 \\
Theorem 1, N=2 & 5.13 \\
Theorem 1, N=3 & 5.74 \\
Theorem 1, N=4 & 5.98 \\
Theorem 1, N=5 & 6.13 \\
Theorem 1, N=6 & 6.16 \\
Theorem 1, N=7 & 6.171 \\
\hline
\end{tabular}

Table 2. The maximal allowable constant delays $\tau$ for Example 2

\section{CONCLUSION}

In this article, the stability of linear systems with constant delays is studied. The parameters which define the LKF are expressed using a solution of a particular linear differential equation which has polynomial solutions. The stability condition is expressed in terms of easy computable LMIs and leads to interesting results in the constant delay case. Even if the discretization method Gu [2001] leads to less conservative conditions, the ones developed in this article are easier to compute. The next step of development would include stability conditions for systems with timevarying delays.

\section{REFERENCES}

Y. Ariba and F. Gouaisbaut. Delay-dependent stability analysis of linear systems with time-varying delay. In Proc. of the $46^{\text {th }}$ IEEE Conference on Decision and Control, New Orleans, LA, USA, 2007a.

Y. Ariba and F. Gouaisbaut. Construction of lyapunovkrasovskii functional for time-varying delay systems analysis. In Proc. of the $47^{\text {th }}$ IEEE Conference on Decision and Control, Cancun, Mexico, 2008.

Y. Ariba and F. Gouaisbaut. Robust stability of timedelay systems with interval delays. In Proc. of the $46^{\text {th }}$ IEEE Conference on Decision and Control, New Orleans, LA, USA, 2007b.

S. Boyd, L. ElGhaoui, and E. Feron. Linear matrix inequalities in system and control theory. SIAM, 1994.

E. Fridman. Descriptor discretized Lyapunov functional method: Analysis and design. IEEE Trans. on Automatic Control, 51(5):890-897, 2006a.

E. Fridman. Stability of systems with uncertain delays: a new "complete" Lyapunov-Krasovskii functional. IEEE Trans. on Automatic Control, 51(5):885 - 890, 2006b.

E. Fridman and S.-I. Niculescu. On complete Lyapunov Krasovskii functional techniques for uncertain systems with fast-varying delays. Int. J. Robust Nonlinear Control, 8(2):364-374, 2008.

E. Fridman and U. Shaked. An improved stabilization method for linear time-delay systems. IEEE Transactions on Automatic Control, 47(11):1931-1937, 2002. 
E. Fridman and U. Shaked. Input-output approach to stability and $L_{2}$-gain analysis of systems with time-varying delays. Systems and Control Letters, 55:10411053, 2006.

E. Fridman, A. Seuret, and J.-P. Richard. Robust sampleddata stabilization of linear systems: An input delay approach. Automatica, 40(8):1141-1446, 2004.

K. Gu. Discretized LMI set in the stability problem of linear uncertain time-delay systems. Int. J. of Control, 68:923 - 934, 1997.

K. Gu. A further refinement of discretized lyapunov functional method for the stability of time-delay systems. Int. J. of Control, 74(10):967 - 976, 2001.

K. Gu and S.-I. Niculescu. Additional dynamincs in transformed time-delay systems. IEEE Trans. Autom. Contr., 45:572-575, 2000.

K. Gu, V.-L. Kharitonov, and J. Chen. Stability of timedelay systems. Birkhauser, 2003.

Y. He, Q. G. Wang, C. Lin, and M. Wu. Further improvement of freeweighting matrices technique for systems with time-varying delay. IEEE Trans. on Automatic Control, 52(2):293299, 2007.

J.P. Hespanha, P. Naghshtabrizi, and Y. Xu. A survey of recent results in networked control systems. Proceedings of the IEEE, 95(1):138-162, 2007.

C. Y. Kao and A. Rantzer. Stability analysis of systems with uncertain time-varying delays. Automatica, 43 959-970, 2007.

C. Y. Kao and A. Rantzer. Robust stability analysis of linear systems with time-varying delays. In 16th IFAC World Congress, IFAC WC'05, Prague, Czech Republic, 2005.

V.B. Kolmanovskii and A.D. Myshkis. Applied theory of functional differential equations. Kluwer, 1999.

V.B. Kolmanovskii and A. Zhabko. Lyapunovkrasovskii approach to the robust stability analysis of time-delay systems. Automatica, 39(1):1520, 2003.

S. Mondie, V.BL. Kharitonov, and O. Santos. Complete lyapunovkrasovskii functional with a given cross term in the time derivative. In Proc. of the $44^{\text {th }}$ IEEE Conference on Decision and Control, Sevilla, Spain, 2005.

P. Naghshtabrizi, J.P. Hespanha, and A.R. Teel. On the robust stability and stabilization of sampled-data systems: A hybrid system approach. In Proceedings of the $45^{\text {th }}$ IEEE Conference on Decision and Control, San Diego, CA, USA, December 13-15, 2006.

S.-I. Niculescu. Delay Effects on Stability. A Robust Control Approach. Springer-Verlag, 2001.

A. Papachristodoulou, M. M. Peet, and S.-I. Niculescu. Stability analysis of linear systems with time-varying delays: Delay uncertainty and quenching. In Proc. of the $46^{t h}$ IEEE Conference on Decision and Control, New Orleans, LA, USA, Dec. 12-14, 2007.

J.-P. Richard. Time delay systems: an overview of some recent advances and open problems. Automatica, 39: 1667-1694, 2003.

A. Seuret and K.H. Johansson. Lyapunov-krasovskii functionals parameterized through linear differential equations. submitted to IEEE Trans. on Automatic Control, 2009.

M. Wu, Y. He, J. H. She, , and G. P. Liu. Delaydependent criteria for robust stability of time-varying delay systems. Automatica, 40(8):14351439, 2004.
V.L. Kharitonov . A.P. Zhabko. Lyapunov-Krasovskii approach to robust stability of time delay systems. In In First IFAC/IEEE symposium on system structure and control, Prague, Rep Tcheque, 2001. 